University of South Carolina

Scholar Commons

\title{
Reduction of Model Order Based on Proper Orthogonal Decomposition for Lithium-Ion Battery Simulations
}

\author{
Long Cai \\ University of South Carolina, cailong2005@gmail.com \\ Ralph E. White \\ University of South Carolina - Columbia, white@cec.sc.edu
}

Follow this and additional works at: https://scholarcommons.sc.edu/eche_facpub

Part of the Other Chemical Engineering Commons

\section{Publication Info \\ Published in Journal of the Electrochemical Society, Volume 156, Issue 3, 2009, pages A154-A161. \\ (c) The Electrochemical Society, Inc. 2009. All rights reserved. Except as provided under U.S. copyright law, this work may not be reproduced, resold, distributed, or modified without the express permission of The Electrochemical Society (ECS). The archival version of this work was published in Cai, L. \& White, R.E. (2009). Reduction of Model Order Based on Proper Orthogonal Decomposition for Lithium-Ion Battery Simulations. Journal of the Electrochemical Society, 156(3): A154-A161 Publisher's Version: http://dx.doi.org/[doi link]} accepted for inclusion in Faculty Publications by an authorized administrator of Scholar Commons. For more information, please contact digres@mailbox.sc.edu. 


\title{
Reduction of Model Order Based on Proper Orthogonal Decomposition for Lithium-Ion Battery Simulations
}

\author{
Long Cai* and Ralph E. White ${ }^{* *, z}$
}

Department of Chemical Engineering, University of South Carolina, Columbia, South Carolina 29208, USA

\begin{abstract}
A reduced-order model (ROM) is developed using proper orthogonal decomposition (POD) for a physics-based lithium-ion battery model. The methodology to obtain the proper orthogonal modes and to analyze their optimality is included. The POD-based ROM for a lithium-ion battery is used to simulate a charge/discharge process and the behavior of a battery pack. Compared to the physics-based model, the computational time to solve the ROM is significantly less, and the two models show excellent agreement.

(C) 2008 The Electrochemical Society. [DOI: 10.1149/1.3049347] All rights reserved.
\end{abstract}

Manuscript submitted July 10, 2008; revised manuscript received November 3, 2008. Published December 30, 2008.

Doyle et al. ${ }^{1}$ and Fuller et al. ${ }^{2}$ published a physics-based model for a lithium-ion cell, which has been used or modified by others. ${ }^{3-7}$ However, these models require significant computation time, which prevents one from using them for control purposes, for example. Consequently, several simplifications of their model have been published to reduce the computation time associated with diffusion of lithium ions in the solid phase. ${ }^{8-10}$ Also, Subramanian et al. ${ }^{11}$ developed a real-time simulation model using a combination of perturbation techniques, volume averaging, and intuition-based simplications. Although they reported that the computational time for their real-time simulation model for a single process was around $100 \mathrm{~ms}$, to derive the lower-order model by using this method one needs to carry out preprocessing and have a priori knowledge of the behavior of the system under different conditions, which makes their method less flexible than desired. Other methods have also been used to derive reduced-order models (ROMs) for lithium-ion batteries, including Chebyshev polynomial methods ${ }^{12}$ and a residue grouping method. ${ }^{13}$ In the methods using Chebyshev polynomials, the state variables are approximated by linear combinations of several Chebyshev polynomials, and then an approximate model is projected onto a subspace formed by these orthonormal Chebyshev polynomials to form an ROM, which can then be solved for the unknown coefficients in the truncated expressions. Smith et al. ${ }^{13}$ developed a control-oriented one-dimensional (1D) electrochemical model by using the method of residue grouping. Their transfer functions are represented by a truncated series of grouped residues with similar eigenvalues.

In this paper, an efficient, systematic, and flexible method known as proper orthogonal decomposition (POD) is introduced for modelorder reduction in lithium-ion battery simulations. This method can be applied in a systematic manner and is flexible. The POD method was introduced by Kosambi, ${ }^{14}$ Loève, ${ }^{15}$ Karhunen, ${ }^{16}$ Pougachev, ${ }^{17}$ and Obukhov ${ }^{18}$ individually and is commonly known as the Karhunen-Loève decomposition method and by other names such as principal components analysis and empirical orthogonal functions analysis in different disciplines such as studies of turbulent flow, ${ }^{19}$ image processing, ${ }^{20}$ signal analysis, ${ }^{21}$ and oceanography. ${ }^{22}$ The method of snapshots ${ }^{23}$ presents an efficient way to determine the proper orthogonal functions, which makes POD a widely used choice in various fields. This paper is organized as follows. The theory and methodology of POD are illustrated in the next section. Then, the procedure is demonstrated by considering lithium ion diffusion in a spherical particle. Finally, POD is used to develop a ROM for the lithium-ion battery model, ${ }^{1,2}$ and simulations are presented.

\footnotetext{
* Electrochemical Society Student Member.

** Electrochemical Society Fellow.

${ }^{\mathrm{z}}$ E-mail: white@engr.sc.edu
}

\section{POD}

POD is a procedure to find a basis for a modal decomposition of an ensemble of signals. Mathematically, this corresponds to finding this basis or set of eigenfunctions in Hilbert space $L^{2}$, which satisfies the following constrained optimization problem ${ }^{19}$

$$
\max _{\phi \in L^{2}}\left\langle|(u, \phi)|^{2}\right\rangle \text { subject to }(\phi, \phi)=1
$$

where $u(x)$ is the ensemble of signals and $\phi(x)$ are the basis functions where $\phi(x) \in L^{2}[\Omega]$ and $x \in \Omega$. $\langle\cdot\rangle$ denotes the time average and $|\cdot|$ the modulus. The inner product of $u$ and $\phi$ in the $L^{2}$ space is defined by $(u, \phi)=\int_{\Omega} u(x) \phi^{*}(x) d x$, where the superscript $*$ refers to the complex conjugate of $\phi(x)$. The constraint imposed on Eq. 1 merely serves to normalize the functions $\phi(x)$ and thus ensure the uniqueness of the solution. The problem defined in Eq. 1 is equivalent to maximizing the functional

$$
L(\phi, \lambda)=\left\langle|(u, \phi)|^{2}\right\rangle-\lambda[(\phi, \phi)-1]
$$

where $\lambda$ is the Lagrange multiplier. Extrema of the functional $L(\phi, \lambda)$ can be found by setting its Gateaux derivative to zero ${ }^{19}$

$$
\left.\frac{\partial}{\partial \delta} L(\phi+\delta \psi, \lambda)\right|_{\delta=0}=0
$$

where $\delta$ is a real number and $\psi$ is an arbitrary function in $L^{2}$ space. Solving Eq. 3 leads to

$$
\int_{\Omega}\left\langle u(x) u^{*}(y)\right\rangle \phi(y) d y=\lambda \phi(x)
$$

The problem defined in Eq. 4 is an infinite dimensional eigenvalue problem. The kernel of the eigenvalue problem is

$$
K(x, y)=\left\langle u(x) u^{*}(y)\right\rangle, \quad \text { where } x, y \in \Omega
$$

which is positively defined and bounded. $K(x, y)$ is also known as the two-point correlation tensor. Equation 4 can be rewritten more compactly as

$$
K \phi=\lambda \phi
$$

According to the Hilbert-Schmidt theorem, ${ }^{24}$ there exists a diagonal decomposition of kernel $K(x, y)$ which is given by

$$
K(x, y)=\sum_{i=1}^{\infty} \lambda_{i} \phi_{i}(x) \phi_{i}^{*}(y)
$$

where $\phi_{i}(x)$ and $\lambda_{i}$ are the eigenfunction/eigenvalue pair of the kernel $K$ and $\lambda_{i} \geqslant 0$. This set of eigenfunctions, $\left\{\phi_{i}(x)\right\}_{i=1}^{\infty}$, forms a complete orthonormal basis in $L^{2}$ space. Every member of the ensemble may be reproduced by a modal decomposition using these eigenfunctions 


$$
u(x)=\sum_{i=1}^{\infty} a_{i} \phi_{i}(x)
$$

The magnitude of the kernel $K$ in some sense denotes the total energy of the dynamic system. ${ }^{19}$ We substitute the infinite series expression of the kernel defined in Eq. 7 in the definition of the norm in $L^{2}$ space. The square norm of $K$ can be determined by

$$
\|K(x, y)\|^{2}=\int_{\Omega} \int_{\Omega}|K(x, y)|^{2} d x d y=\sum_{i=1}^{\infty} \lambda_{i}^{2}
$$

Equation 9 indicates that the total energy of the ensemble is the sum of the square of the eigenvalues of $K$. Every eigenvalue indicates the importance of the corresponding eigenfunction to representation of the ensemble. Consequently, we can sort the eigenvalue/ eigenfunction pair by $\lambda_{i} \geqslant \lambda_{i+1}$ and choose the first $N$ eigenfunctions to form a subspace $\left\{\phi_{i}(x)\right\}_{i=1}^{N}$. The projection of the signal $u(x, t)$ of the ensemble onto this subspace is given by

$$
\hat{u}(x, t)=\sum_{i=1}^{N} a_{i}(t) \phi_{i}(x)
$$

The optimality of the POD which is verified in Ref. 19 and 24 states that

1. The POD coefficients $a_{i}(t)$ are uncorrelated, so that $\left\langle a_{i}(t) a_{j}^{*}(t)\right\rangle=\delta_{i j} \lambda_{i}$.

2. The infinite set of functions $\left\{\psi_{i}(x)\right\}_{i=1}^{\infty}$ can be an arbitrary orthonormal set such that $u(x, t)=\sum_{i=1}^{\infty} b_{i}(t) \psi_{i}(x)$. For every $N$ we have $\sum_{i=1}^{N}\left\langle a_{i}(t) a_{i}^{*}(t)\right\rangle=\sum_{i=1}^{N} \lambda_{i} \geqslant \sum_{i=1}^{N}\left\langle b_{i}(t) b_{i}^{*}(t)\right\rangle$.

The second statement is the basis for the claim that the POD is optimal for modeling or reconstructing a signal $u(x, t)$. It implies that among all linear decompositions, Eq. 10 is the most compact in the sense that, for a given number of modes $(N)$, the projection on the subspace $\left\{\phi_{i}(x)\right\}_{i=1}^{N}$ will contain the most "kinetic energy" on average. For convenience, we name the POD coefficients $a_{i}(t)$ the reduced variables associated with $u(x, t)$ and the elements in the subspace $\phi_{i}(x)$ the proper orthogonal modes (POMs).

For the cases where the analytical solutions for $\phi(x)$ cannot by solved from Eq. 6, a numerical scheme is required. A data ensemble $\mathrm{Y}$ can be prepared from a set of vector valued signals denoted by $y_{j}=u(\underline{x}, j \tau) \in \mathbb{R}^{m}$ from the solution of $u$ evaluated on the $m$ discrete spatial node points $\underline{x}$ at the $j$ th time step $(j \tau)$ and is an $m$ $\times n$ matrix, $\mathrm{Y}=\left[y_{1}, \underline{y}_{2}, \ldots, \underline{y}_{n}\right]$, where $n$ is the total number of time steps. The discrete form of the kernel defined in Eq. 5 is given by ${ }^{25}$

$$
\bar{K}=\frac{1}{n} Y^{\mathrm{T}}
$$

where $\mathrm{Y}^{\mathrm{T}}$ is the transpose of $\mathrm{Y}$. A singular value decomposition (svd) of the discrete kernel $\bar{K}$ yields

$$
\operatorname{svd}(\bar{K})=\Phi \Sigma \Phi^{\mathrm{T}} \text { or } \mathrm{YY}^{\mathrm{T}} \Phi=\Phi \Lambda
$$

where $\Lambda=\Sigma / n$. In Eq. 12, $\Phi$ is an $m \times m$ matrix, and $\Sigma$ is $m \times m$ diagonal matrix. The nonzero diagonal elements in $\Sigma$ are on the order of $\sigma_{1} \geqslant \sigma_{2}, \ldots, \geqslant \sigma_{d}>0$, where $d=\min (m, n)\left(\lambda_{i}=\sigma_{i} / n\right)$. Column vectors in $\Phi$ form an orthonormal basis and are the POMs in $\mathbb{R}^{m}$ space.

The diagonal decomposition defined in Eq. 12 is an $m$-order eigenvalue problem. If the number of spatial node points is large, it is difficult to determine the POMs by solving Eq. 12. If $m>n$ (for instance, in the pseudo-two-dimensional (2D) diffusion equation of the lithium-ion battery model, $m=5000$ and $n$ is about tens to hundreds), it is more convenient to convert the $m$-order eigenproblem to an $n$-order eigenproblem if the number of time steps $n$ is much less than $m$. The method of snapshots ${ }^{23}$ provides an efficient way to conduct this conversion. The solution for the signal at each time step is arranged as a vector and named a snapshot. The ensemble $\mathrm{Y}$ is a collection of these snapshots. The main idea of the method of snapshots is that the POMs are the linear combination of the snapshots

$$
\Phi=\mathrm{YA}
$$

where the ensemble $\mathrm{Y}$ is an $m \times n$ matrix, $m$ is the number of node points, $n$ is the number of snapshots, and the matrix A remains to be determined. Substitution of Eq. 13 into Eq. 12 yields an $n$ th-order eigenvalue problem

$$
\mathrm{Y}^{\mathrm{T}} \mathrm{YA}=\mathrm{A} \Lambda
$$

where $A$ is the eigenvectors of transformation $\mathrm{Y}^{\mathrm{T}} \mathrm{Y}$. Finally, the POMs are determined by normalizing the column vector $\phi_{j}$ of $\Phi$ by its norm

$$
\phi_{j}=\frac{\phi_{j}}{\left\|\phi_{j}\right\|}, \quad j=1, \ldots, n
$$

\section{POD-based ROM}

The POD-based ROM can be derived from a physics-based model, which consists of a set of partial (temporal-spatial) differential equations, by the following five steps:

1. Formulate the discrete model (rigorous model) using a spatial discretization method for the physical model. The resulting system of differential and algebra equations (DAEs) is referred to as the rigorous model.

2. Solve the rigorous model and sample the solution at a given rate to form a data ensemble Y. Decompose the discrete kernel, $\bar{K}$, constructed from the data ensemble, Y, using singular value decomposition method. The POMs, $\phi_{i}(x)$, are the resulting eigenvectors which are sorted in a manner such that the corresponding eigenvalues are in a nonincreasing order.

3. Chose the first $N$ POMs $\phi_{i}(x)$ to form a subspace. Approximate the discrete field variables in the rigorous model by the linear combination of the first $N$ of the POMs, each of which is multiplied by a time-dependent reduced variable, $a_{i}(t)$.

4. Substitute the approximation of the state variables into the rigorous model, and project the resulting system onto the subspace to obtain the ROM.

5. Solve the ROM for the reduced variables. Reconstruct the original discrete field variables by using step 3 again.

We illustrate the procedure to derive the POD-based ROM by considering lithium-ion diffusion in a spherical particle. The governing equation for lithium-ion diffusion in a spherical particle is given by the second Fick's law

$$
\frac{\partial c_{\mathrm{s}}(r, t)}{\partial t}=D_{\mathrm{s}} \frac{1}{r^{2}} \frac{\partial}{\partial r}\left[r^{2} \frac{\partial c_{\mathrm{s}}(r, t)}{\partial r}\right]
$$

with the boundary conditions

$$
\begin{gathered}
-\left.D_{\mathrm{s}} \frac{\partial c_{\mathrm{s}}(r, t)}{\partial r}\right|_{r=0}=0 \\
-\left.D_{\mathrm{s}} \frac{\partial c_{\mathrm{s}}(r, t)}{\partial r}\right|_{r=R_{s}}=j(t)
\end{gathered}
$$

and the initial condition

$$
c_{\mathrm{s}}(r, 0)=c_{\mathrm{s}, 0}
$$

where $D_{\mathrm{s}}$ is the diffusion coefficient of lithium ion in the solid phase, $R_{\mathrm{S}}$ is the radius of the particle, and $j(t)$ is the pore-wall flux of the lithium ions. The discrete model is derived using the finitevolume method. The geometry is partitioned uniformly into $m$ finite volumes as shown in Fig. 1. Every finite volume is enclosed by two surfaces symboled by $w$ on the west side and $e$ on the east side, respectively. Every node point is located at the center of the corresponding volume element. The node point of interest is assigned a capital letter $\mathrm{P}$; node point $\mathrm{W}$ is the left neighbor of $\mathrm{P}$ and node point 




Figure 1. The finite volume method schematic in the 1D diffusion problem.

$\mathrm{E}$ is the right neighbor. The size of the Pth control volume is represented by $h_{\mathrm{P}}, c_{s, \mathrm{P}}$ is the concentration at node point $\mathrm{P}$, and $c_{s, \mathrm{~L}}$ and $c_{s, \mathrm{R}}$ are the concentration on the left and right boundary, respectively. The discrete model is obtained from Eq. 16-19.

\begin{tabular}{lcc}
\hline \multicolumn{2}{l}{ Table I. Parameters in the diffusion system. } \\
Parameter & Value & Unit \\
\hline$a_{\mathrm{s}}$ & $1.74 \times 10^{6}$ & $\mathrm{~m}^{-1}$ \\
$c_{s, 0}$ & 46404.0 & $\mathrm{~mol} / \mathrm{m}^{3}$ \\
$D \mathrm{~s}$ & $2.0 \times 10^{-16}$ & $\mathrm{~m}^{2} / \mathrm{s}$ \\
$h_{\mathrm{P}}$ & $R_{\mathrm{s}} / n$ & $\mathrm{~m}$ \\
$I_{0}$ & $1.334 \times 10^{5}$ & $\mathrm{~A} / \mathrm{m}^{3}$ \\
$l_{1}$ & $0.5 h_{\mathrm{P}}$ & $\mathrm{m}$ \\
$l_{2}$ & $1.5 h_{\mathrm{P}}$ & $\mathrm{m}$ \\
$m$ & 100 & \\
$n$ & 201 & $\mathrm{~m}$ \\
$R_{\mathrm{s}}$ & $1.0 \times 10^{-6}$ &
\end{tabular}

at $5 \mathrm{~s}$ intervals starting at $0 \mathrm{~s}$ ). The POMs are calculated using Eq. 12 and the first seven vector-valued POMs $\phi_{i}(r)$ are plotted in Fig. 2.

The importance of each POM to the system is denoted by the value of the corresponding eigenvalue. The POMs obtained by svd

$$
\begin{cases}\frac{d c_{\mathrm{s}, \mathrm{P}}}{d t}=\frac{D_{\mathrm{s}, \mathrm{e}}}{\frac{1}{2} h_{\mathrm{P}}\left(h_{\mathrm{P}}+h_{\mathrm{E}}\right)} \frac{r_{\mathrm{e}}^{2}}{r_{\mathrm{P}}^{2}}\left(c_{\mathrm{s}, \mathrm{E}}-c_{\mathrm{s}, \mathrm{P}}\right), & \mathrm{P}=1 \\ \frac{d c_{\mathrm{s}, \mathrm{P}}}{d t}=\frac{D_{\mathrm{s}, \mathrm{e}}}{\frac{1}{2} h_{\mathrm{P}}\left(h_{\mathrm{P}}+h_{\mathrm{E}}\right)} \frac{r_{\mathrm{e}}^{2}}{r_{\mathrm{P}}^{2}}\left(c_{\mathrm{s}, \mathrm{E}}-c_{\mathrm{s}, \mathrm{P}}\right)-\frac{D_{\mathrm{s}, \mathrm{W}}}{\frac{1}{2} h_{\mathrm{P}}\left(h_{\mathrm{P}}+h_{\mathrm{W}}\right)} \frac{r_{\mathrm{w}}^{2}}{r_{\mathrm{P}}^{2}}\left(c_{\mathrm{s}, \mathrm{P}}-c_{\mathrm{s}, \mathrm{W}}\right), & \mathrm{P}=2, \ldots, m-1 \\ \frac{d c_{\mathrm{s}, \mathrm{P}}}{d t}=-\frac{r_{\mathrm{e}}^{2}}{h_{\mathrm{P}} r_{\mathrm{P}}^{2} j(t)-\frac{D_{\mathrm{s}, \mathrm{W}}}{\frac{1}{2} h_{\mathrm{P}}\left(h_{\mathrm{P}}+h_{\mathrm{W}}\right)} \frac{r_{\mathrm{w}}^{2}}{r_{\mathrm{P}}^{2}}\left(c_{\mathrm{s}, \mathrm{P}}-c_{\mathrm{s}, \mathrm{W}}\right),} & \mathrm{P}=m\end{cases}
$$

where $\mathrm{W}=\mathrm{P}-1, \mathrm{E}=\mathrm{P}+1$, and the locations of $r_{e}, r_{w}$, and $r_{\mathrm{P}}$ are shown in Fig. 1. Equation 20 can be written in the matrix notation as

$$
\begin{gathered}
\dot{C}=\mathrm{A} \cdot \underline{C}+\underline{b} j(t) \\
C(0)=\underbrace{\left[c_{\mathrm{s}, 0}, \ldots, c_{\mathrm{s}, 0}\right]^{\mathrm{T}}}_{\mathrm{m}}
\end{gathered}
$$

where $\underline{C}=\left[c_{\mathrm{s}, 1}, c_{\mathrm{s}, 2}, \ldots, c_{\mathrm{s}, \mathrm{m}}\right]^{\mathrm{T}}$ and $\underline{b}=\left[0 \ldots-r_{\mathrm{e}}^{2} /\left(h_{\mathrm{m}} r_{\mathrm{m}}^{2}\right)\right]^{\mathrm{T}}$. The concentrations on the boundaries can be obtained after solving Eq. 20 from the discretized boundary conditions by means of the threepoint forward finite-difference approximation for the concentration derivative on the left end and three-point backward finite difference on the right end, respectively, and given by

$$
\begin{aligned}
c_{\mathrm{s}, \mathrm{L}} & =\frac{l_{2}^{2} c_{\mathrm{s}, 1}-l_{1}^{2} c_{\mathrm{s}, 2}}{\left(l_{2}^{2}-l_{1}^{2}\right)} \text { and } c_{\mathrm{s}, \mathrm{R}} \\
& =\frac{-l_{1} l_{2}\left(l_{2}-l_{1}\right) j(t) /\left(a_{\mathrm{s}} F\right)+D_{\mathrm{s}} l_{2}^{2} c_{\mathrm{s}, \mathrm{m}}-D_{\mathrm{s}} l_{1}^{2} c_{\mathrm{s}, \mathrm{m}-1}}{D_{s}\left(l_{2}^{2}-l_{1}^{2}\right)}
\end{aligned}
$$

In Eq. 22, the values for $l_{1}$ and $l_{2}$ are defined in Fig. 1 .

Next, the discrete model with the constant flux on the surface, $j(\mathrm{t})=I_{0} /\left(a_{\mathrm{s}} F\right)$, is defined in Eq. 20 in the time range 0-1000 s based on the values of the model parameters given in Table I. The solution is used to generate the data ensemble $\mathrm{Y}$ by creating 100 rows (one row for each node point) and 201 columns (each column of the discrete kernel are sorted automatically in such an order that the magnitudes of the corresponding eigenvalues decrease with the index of the POMs. The eigenvalues are presented in Fig. 3. The magnitudes decrease sharply for the first 12 eigenvalues, and they do not change significantly and are tiny when $i$ is greater than 12 . We draw a vertical line at $i=13$. Those points to the left side of the vertical line reveal that their corresponding POMs are significant, and those to the right of $i=13$ are not. Bearing this in mind, we approximate the discrete concentration $\underline{C}$ by a linear combination of the first $N(N \leqslant 12)$ POMs, that is

$$
\underline{C}(t)=\Phi \underline{a}(t)
$$

where $\Phi$ consists of the first $N(N \leqslant 12)$ POMs and $a(t)$ $=\left[a_{1}(t), a_{2}(t), \ldots, a_{N}(t)\right]^{\mathrm{T}}$ are the reduced variables. There is no theoretical criterion to determine the value of $N$. Generally, $N$ is chosen as the minimum value to satisfy the following inequality

$$
E C_{N}=\sum_{i=1}^{N} \lambda_{i} / \sum_{i=1}^{100} \lambda_{i} \geqslant 99.99 \%
$$

where $E C_{N}$ is named the energy content of the first $N$ POMs. Substituting the approximation defined in Eq. 23 back into Eq. [21], we obtain

$$
\Phi \underline{\dot{a}}=\mathrm{A} \Phi \underline{a}+\underline{b} j(t)
$$

In Eq. 25, there are 100 equations and $N$ unknowns. We project this overdetermined system onto the subspace $\Phi$ to obtain the ROM with $N$ dimensions 


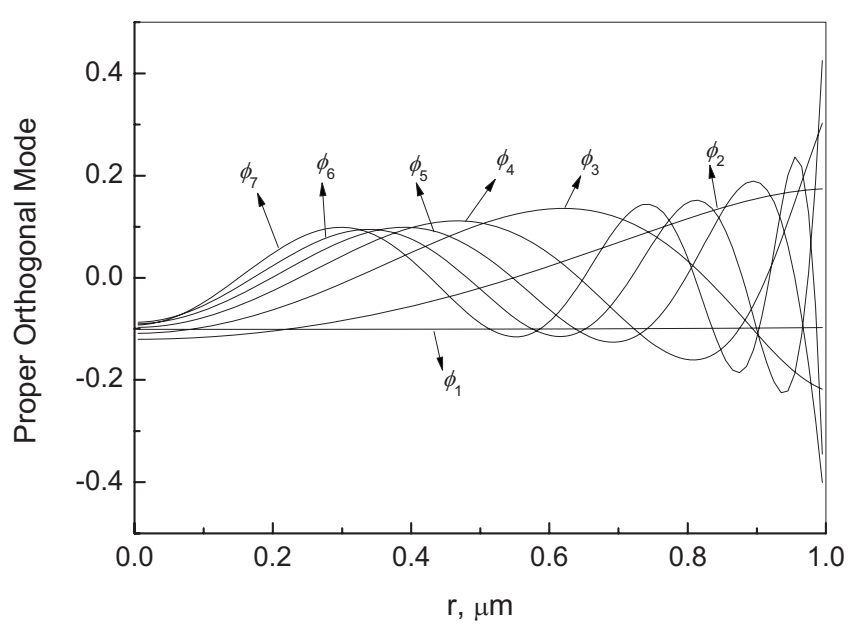

Figure 2. The first seven POMs extracted from the spherical diffusion example with the conditions $j(t)=I_{0} /\left(a_{\mathrm{s}} F\right)$ and $D_{\mathrm{s}}=2.0 \times 10^{-16} \mathrm{~m}^{2} / \mathrm{s}$.

$$
\underline{a}=\Phi^{\mathrm{T}} \mathrm{A} \Phi \underline{a}+\Phi^{\mathrm{T}} \underline{b} j(t)
$$

To derive the above ROM, the orthonormality of the POMs is applied implicitly. The initial conditions for the ROM are determined by

$$
\underline{a}(0)=\Phi \underline{C}(0)
$$

Equations 26 and 27 can be solved for $a$ using an ordinary differential equations solver. The values of the concentration at the node points are obtained by substituting the values of the reduced variables $\underline{a}$ into the approximation defined in Eq. 23. In our simple linear problem, the coefficient matrices A and $\underline{b}$ in Eq. 26 are independent of time and concentration.

The simulation results of the ROM are compared to those of the rigorous model with the pore wall flux of the lithium ions $j(t)$ $=I_{0} /\left(a_{\mathrm{S}} F\right)$ at the five particular times of $0,250,500,750$, and $1000 \mathrm{~s}$, and are presented in Fig. 4. The POMs used in formulating the ROM are extracted from the data ensemble obtained by solving the rigorous model under the same conditions. Figure 4 shows that the ROM agrees well with the rigorous model.

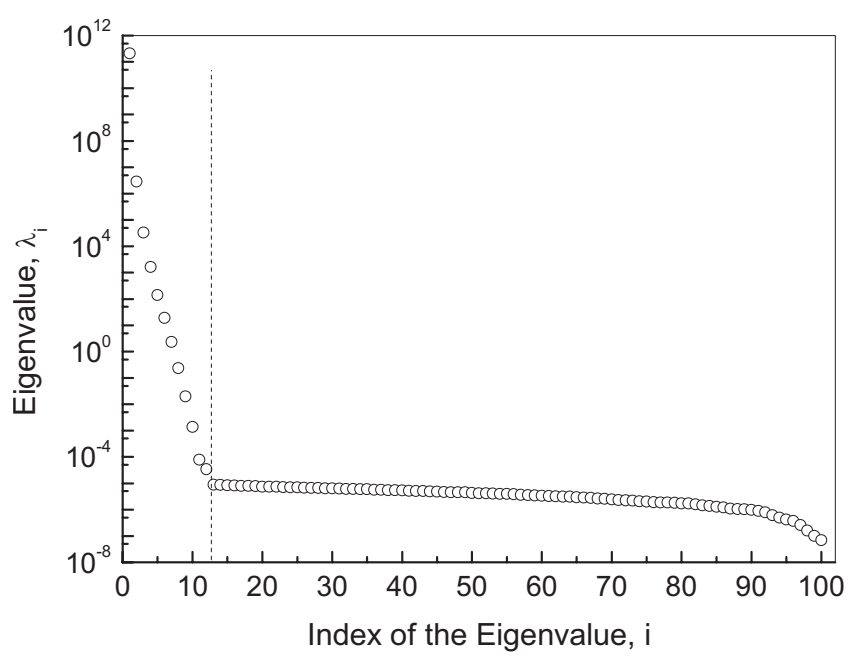

Figure 3. Eigenvalue spectrum obtained from the data ensemble consisting of the discrete-model solution under $j(t)=I_{0} /\left(a_{\mathrm{s}} F\right)$ and $D_{\mathrm{s}}=2.0$ $\times 10^{-16} \mathrm{~m}^{2} / \mathrm{s}$.

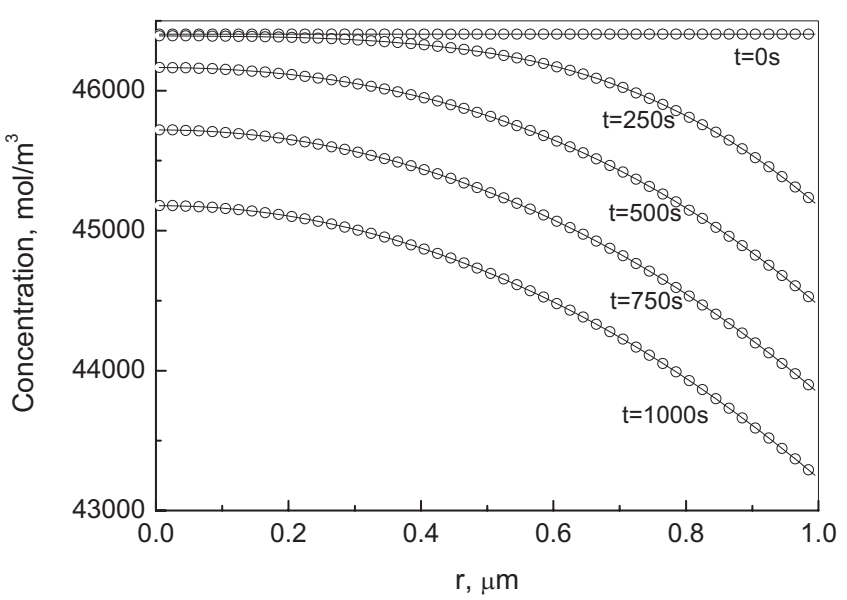

Figure 4. Comparison of the ROM to the rigorous model at $0,250,750$, and $1000 \mathrm{~s}$.

\section{Applications of the POD-based ROM for Lithium-Ion Battery}

The schematic of the $\mathrm{LiMnO}_{2}$-carbon battery is shown in Fig. 5 . From left to right, the components of the battery are aluminum current collector, $\mathrm{LiMnO}_{2}$ positive electrode, separator, carbon negative electrode, and copper current collector. There are four interfaces involved, and these are located at $x=0, x=L_{\mathrm{p}}, x=L_{\mathrm{p}}$ $+L_{\mathrm{s}}$, and $x=L_{\mathrm{p}}+L_{\mathrm{s}}+L_{\mathrm{n}}$, respectively. The governing equations (porous electrode model) and related expressions are summarized in Table II. In the full cell model, the particles in solid phase are divided into 50 control volumes, and the regions of positive electrode, separator, and negative electrode are discretized into 100, 70, and 100 control volumes, respectively. On every node point of the $x$ coordinate, there are $50+1+3$ unknowns (50 unknowns are assigned for concentration of lithium ion in particles, 1 unknown is assigned for concentration on the surface of the particle, and the other 3 unknowns are assigned for concentration in liquid phase, potential in solid phase and potential in liquid phase). This model has been called the pseudo-2D model, ${ }^{1}$ because at a particular $x$ value, diffusion occurs in the solid phase in the $r$ direction only. Consequently, there are 14,580 equations in our high-order dimensional discrete model. Actually, no solid-phase exists in the separator region, but for numerical simulation convenience, we include

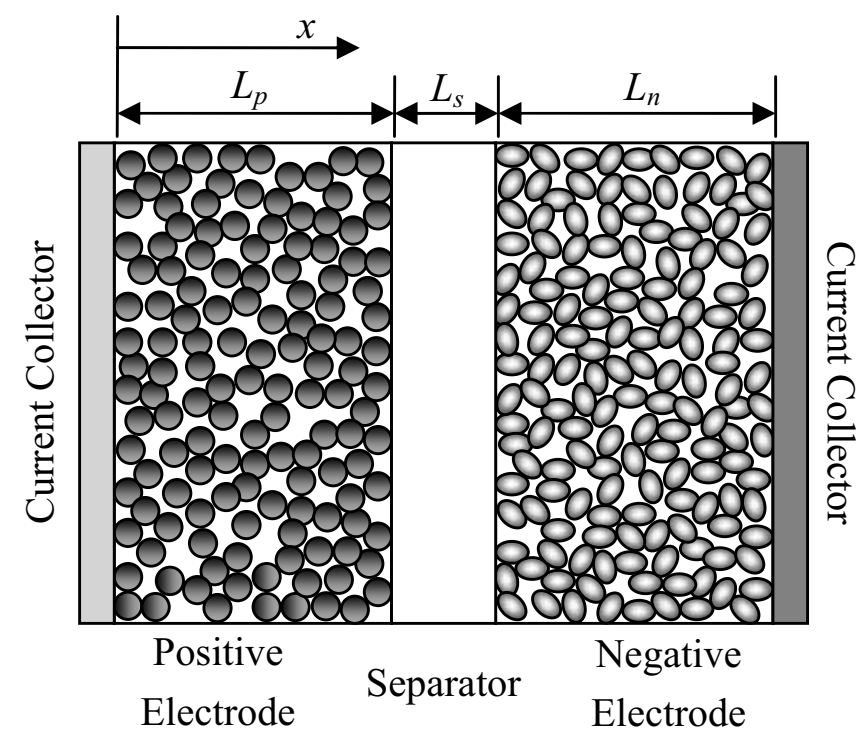

Figure 5. Schematic of a lithium-ion battery. 


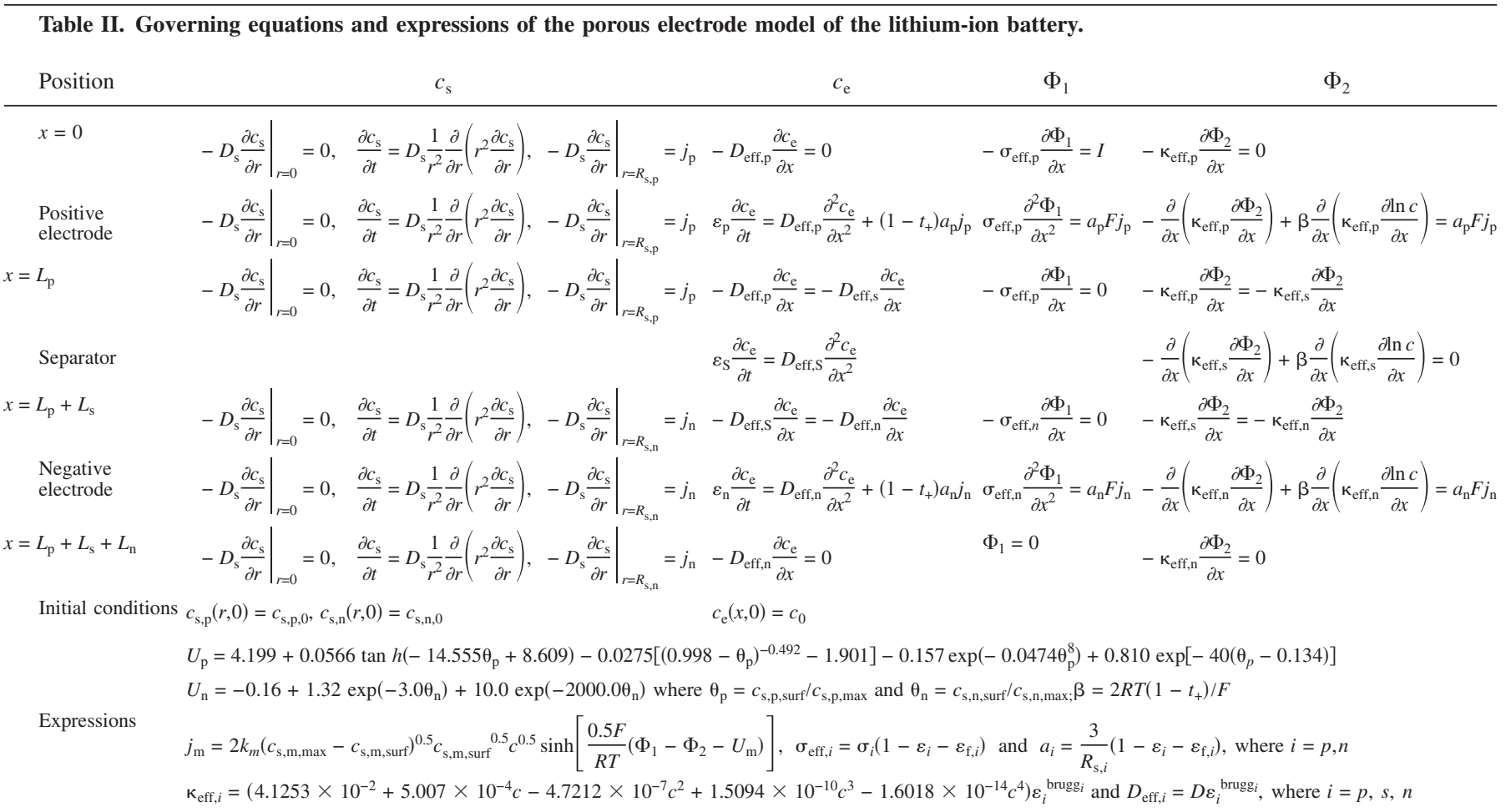

hypothetical solid phase concentration and potential in this region and assign zeros for their values. The rigorous model under $1 \mathrm{C}$ discharge is solved by a DAE solver using the parameters in Table III.

The simulation results are sampled to form a data ensemble for each state variable $\left(c_{\mathrm{s}, \mathrm{p}}, c_{\mathrm{s}, \mathrm{n}}, c_{\mathrm{s}, \mathrm{p} \text {,surf }}, \mathrm{c}_{\mathrm{s}, \mathrm{n}, \mathrm{surf}}, \Phi_{1, \mathrm{p}}, \Phi_{1, \mathrm{n}}, c_{\mathrm{e}}\right.$, and $\left.\Phi_{2}\right)$. The POMs for every state variable are calculated using the procedures mentioned in the previous section. Every state variable has a designated reduced variable array correspondingly named $\underline{a}_{\mathrm{csp}}, \underline{a}_{\mathrm{cs} n}$, $\underline{a}_{\mathrm{cspsurf}}, \underline{a}_{\mathrm{csnsurf}}, \underline{a}_{\Phi 1 \mathrm{p}}, \underline{a}_{\Phi 1 \mathrm{n}}, \underline{a}_{\mathrm{ce}}$, and $\underline{a}_{\Phi 2}$. The dimensions of the reduced variable arrays are determined as $9,9,4,4,4,4,8$, and 8 (total of 50), respectively, to catch an energy content ratio of $99.99 \%$ according Eq. 24. Consequently, there are 50 equations used in the ROM to solve for the 50 reduced variables. Figure $6 \mathrm{a}$ shows the simulated discharge curves at the rate of $0.1,1,2,4,6,10$, and $20 \mathrm{C}$ using this $1 \mathrm{C}$ ROM and compared to the results of the rigorous model. The symbols denote the results of ROM, while the solid lines are the results of the rigorous model. The relative errors of the time at the end of discharge of the ROM are listed in Table IV. The ROM matches well (less than $1 \mathrm{mV}$ difference) to the rigorous model at the low-rate, less than $4 \mathrm{C}$, discharge process, it is $1.3 \%$ in error at $10 \mathrm{C}$ and $25 \%$ at $20 \mathrm{C}$.

Considering that the behavior of the battery varies significantly from low current rates to high current rates, we include the simulation results of the rigorous model at various rates of discharge which range from $0.1 \mathrm{C}$ to $20 \mathrm{C}$ in our data ensemble to compute POMs for the state variables. The simulated discharge curves under various rates ranging from $0.1 \mathrm{C}$ to $20 \mathrm{C}$ by $\mathrm{ROM}$ are plotted in Fig. $6 \mathrm{~b}$. The improved ROM agrees well with the rigorous model within the whole computational range of the current rate, and the relatively ending time errors for this improved ROM are summarized in Table IV. The computational time of the ROM for the $1 \mathrm{C}$ discharge process is around $1.5 \mathrm{~s}$, but to solve the same problem the rigorous model requires around $10 \mathrm{~s}$ on a computer with $2.0 \mathrm{GHz}$ processor and 2.0 GB RAM. We cannot reduce the computational time proportional to the orders reduction of differential/algebraic equations from the rigorous model to ROM, because the coefficient matrix

\section{Table III. Parameters of the model of the lithium-ion battery.}

\begin{tabular}{|c|c|c|c|c|c|}
\hline Parameter & Value & Unit & Parameter & Value & Unit \\
\hline$L_{\mathrm{p}}$ & 183 & $\mu \mathrm{m}$ & $c_{\mathrm{s}, \mathrm{p}, \max }$ & 22,860 & $\mathrm{~mol} / \mathrm{m}^{3}$ \\
\hline$L_{\mathrm{s}}^{\mathrm{P}}$ & 52 & $\mu \mathrm{m}$ & $c_{\mathrm{s}, \mathrm{n}, \max }^{\mathrm{s}, \max }$ & 26,390 & $\mathrm{~mol} / \mathrm{m}^{3}$ \\
\hline$L_{\mathrm{n}}$ & 100 & $\mu \mathrm{m}$ & $c_{\mathrm{s}, \mathrm{p}, 0}$ & 3900 & $\mathrm{~mol} / \mathrm{m}^{3}$ \\
\hline$R_{\mathrm{s}, \mathrm{p}}$ & 8 & $\mu \mathrm{m}$ & $c_{\mathrm{s}, \mathrm{n}, 0}^{\mathrm{s}, \mathrm{O}}$ & 14870 & $\mathrm{~mol} / \mathrm{m}^{3}$ \\
\hline$R_{\mathrm{s}, \mathrm{n}}$ & 12.5 & $\mu \mathrm{m}$ & $\sigma_{p}$ & 3.8 & $\mathrm{~S} / \mathrm{m}$ \\
\hline$D_{\mathrm{s}, \mathrm{p}}^{\mathrm{s}}$ & $1.0 \times 10^{-13}$ & $\mathrm{~m}^{2} / \mathrm{s}$ & $\sigma_{\mathrm{n}}$ & 100 & $\mathrm{~S} / \mathrm{m}$ \\
\hline$D_{\mathrm{s}, \mathrm{n}}^{\mathrm{s}, \mathrm{p}}$ & $3.9 \times 10^{-14}$ & $\mathrm{~m}^{2} / \mathrm{s}$ & Brugg $_{p}$ & 1.5 & - \\
\hline$D_{\mathrm{e}}^{, 11}$ & $7.5 \times 10^{-11}$ & $\mathrm{~m}^{2} / \mathrm{s}$ & Brugg $_{s}$ & 1.5 & - \\
\hline$\varepsilon_{\mathrm{p}}$ & 0.444 & - & Brugg $_{n}$ & 1.5 & - \\
\hline$\varepsilon_{\mathrm{s}}$ & 1.0 & - & $k_{\mathrm{p}}$ & $2.334 \times 10^{-11}$ & $\mathrm{~mol} / \mathrm{m}^{2} \mathrm{~s} /\left(\mathrm{mol} / \mathrm{m}^{3}\right)^{1.5}$ \\
\hline$\varepsilon_{\mathrm{n}}$ & 0.357 & - & $k_{\mathrm{n}}$ & $2.334 \times 10^{-11}$ & $\mathrm{~mol} / \mathrm{m}^{2} \mathrm{~s} /\left(\mathrm{mol} / \mathrm{m}^{3}\right)^{1.5}$ \\
\hline$\varepsilon_{\mathrm{f,p}}$ & 0.259 & - & $t_{+}$ & 0.363 & - \\
\hline$\varepsilon_{\mathrm{f}, \mathrm{p}}$ & 0.172 & - & $I$ & 17.5 (1C rate) & $\mathrm{A} / \mathrm{m}^{2}$ \\
\hline$c_{0}$ & 2000 & $\mathrm{~mol} / \mathrm{m}^{3}$ & $T$ & 298 & K \\
\hline
\end{tabular}




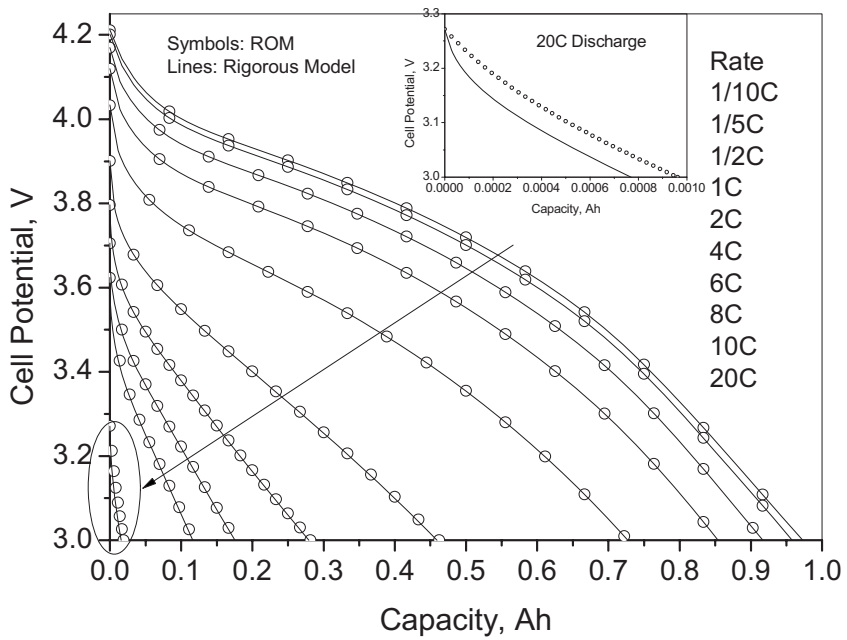

a

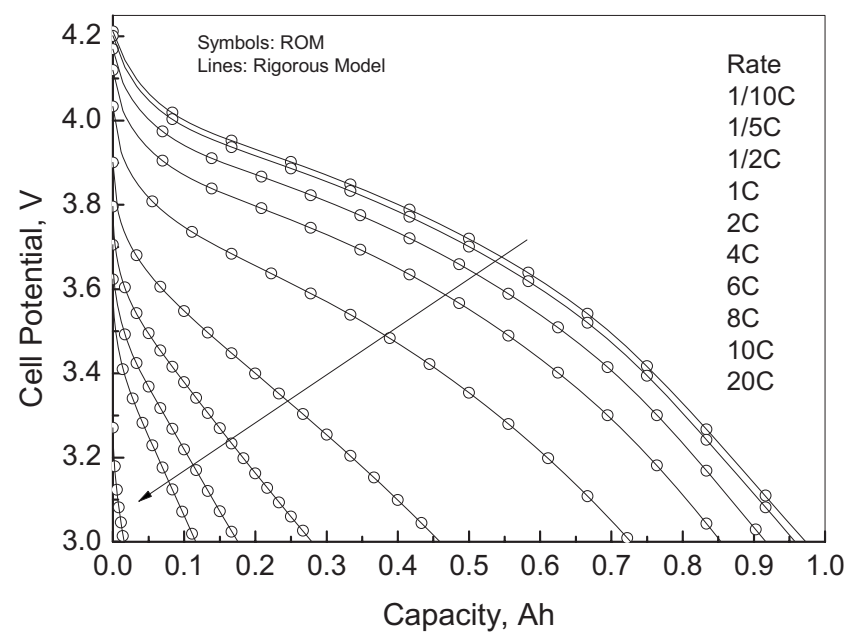

b

Figure 6. Comparison of (a) the ROM and (b) the improved ROM to the rigorous model for the discharge simulations under $0.1,1,2,4,6,8,10$, and $20 \mathrm{C}$.

$\Phi^{\mathrm{T}} \mathrm{A} \Phi$ in Eq. 26 is evaluated and the state variables are reconstructed at every iteration due to the nonlinear and coupled nature of the system. The lithium-ion concentrations in the liquid phase at the four interfaces mentioned above as functions of time were computed using the ROM and the rigorous model under two different discharge rates. $1 \mathrm{C}$ and $10 \mathrm{C}$, and are plotted in Fig. 7a and b, respectively. The symbols denote the results of the ROM, while the solid
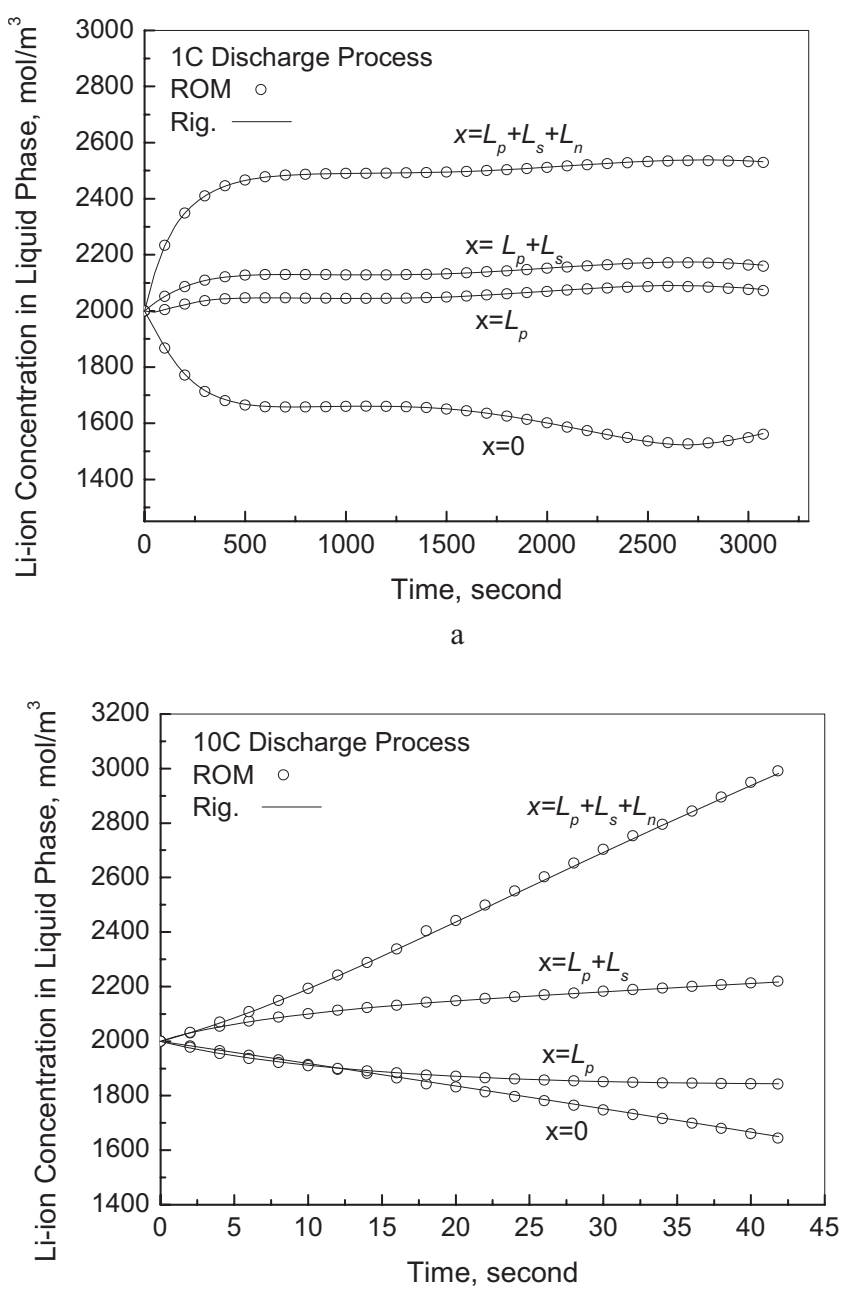

b

Figure 7. Comparison of the concentrations of lithium ion in the liquid phase at the four specified interfaces obtained from the improved ROM to those by rigorous model under (a) $1 \mathrm{C}$, and (b) $10 \mathrm{C}$ rate discharge.

lines are the results of the rigorous model. Figures $7 \mathrm{a}$ and $\mathrm{b}$ indicate that the ROM captures the dominant properties of the system and agrees well with the rigorous model not only under low rate conditions but also during discharge rates as high as 10C.

The results of cycling simulations are shown in Fig. 8. The battery is discharged first at a $1 \mathrm{C}$ rate until $3.0 \mathrm{~V}$, followed by a $1 \mathrm{C}$ charge process up to $4.3 \mathrm{~V}$, and then the battery is charged at $4.3 \mathrm{~V}$ until the current decreases to $10 \mathrm{~mA}$. At the end of the cycling, the stopping times are 18,291.6 and 18,307.2 $\mathrm{s}$ for the rigorous model

\section{Table IV. Relative error of ending time of the ROM.}

\begin{tabular}{|c|c|c|c|}
\hline $\begin{array}{l}\text { Current rate } \\
\text { (C) }\end{array}$ & $\begin{array}{l}\text { Discharge ending } \\
\text { time for the rigorous } \\
\text { model (s) }\end{array}$ & $\begin{array}{l}\text { Discharge ending-time } \\
\text { error for the ROM based on the } 1 \mathrm{C} \\
\text { data ensemble }(\%)\end{array}$ & $\begin{array}{l}\text { Discharge ending-time } \\
\text { error for the ROM based on the multirates } \\
\text { data ensemble }(\%)\end{array}$ \\
\hline 0.1 & 35012.0 & $2.8 \times 10^{-4}$ & 0.09 \\
\hline 1 & 3073.69 & $3.0 \times 10^{-4}$ & 0.016 \\
\hline 2 & 1308.59 & 0.026 & $1.5 \times 10^{-3}$ \\
\hline 4 & 413.462 & 0.69 & 0.06 \\
\hline 6 & 167.701 & 0.81 & 0.11 \\
\hline 8 & 78.9494 & 0.86 & 0.06 \\
\hline 10 & 41.8910 & 1.30 & 0.14 \\
\hline 20 & 2.77001 & 25.0 & 1.30 \\
\hline
\end{tabular}




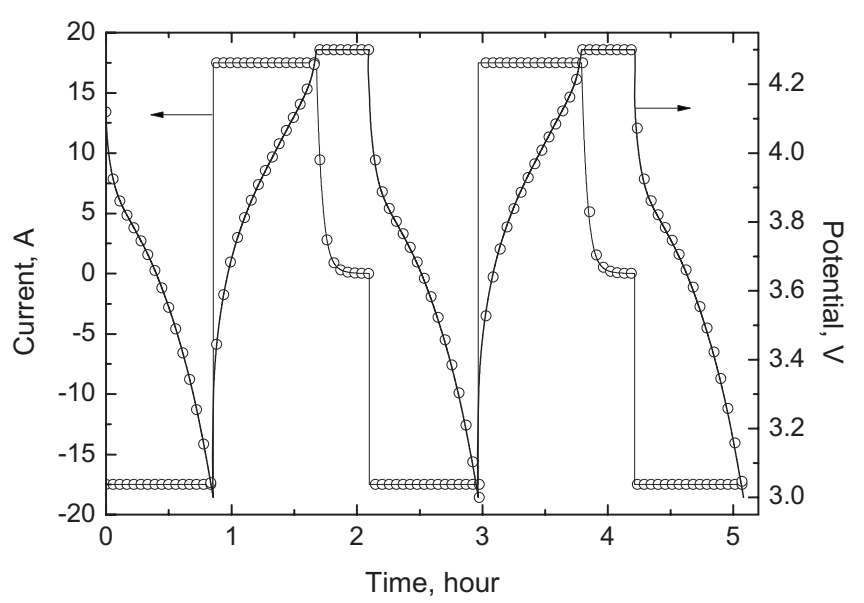

Figure 8. Cell-cycling simulation results based on the ROM (denoted by symbols) compared to results of the rigorous model (denoted by solid lines). (Cell-cycling protocol: $1 \mathrm{C}$ discharge to $3.0 \mathrm{~V}$, followed by $1 \mathrm{C}$ charge to $4.3 \mathrm{~V}$, and then charge the cell holding the cell potential at $4.3 \mathrm{~V}$ until the current decreases to $10 \mathrm{~mA}$ ).

and the improved ROM, respectively, and the relative difference is $0.85 \%$. A major part of this difference arises from the constantvoltage charge step. We could further improve the accuracy of the ROM by including additional high-order simulation results into the snapshot case.

The more-attractive application of ROM is the simulation of battery packs, as the simulation of battery packs using the rigorous model would require significant computation time because the coefficient matrix is no longer banded. If we treat this unbanded matrix as a full matrix in the DAE solver, the computational time increases dramatically. Moreover, the computer resources needed to store the state variables exceed the capacity of the DAE solver on current PCs, which makes it impossible to run battery-pack simulations using the rigorous model. Figure 9 shows the simulated individual currents across four cells in parallel using the improved ROM with 200 reduced variables (50 for each cell). The four cells are identical except for the difference in the initial states of charge and are discharged at $0.5 \mathrm{C}$ rate. The values of the initial states of charges are chosen arbitrarily, which illustrates the capability of POD-based ROM to simulate the behavior of a battery pack.



Figure 9. Currents across the individual cells of a battery with four cells in parallel during discharge at the $0.5 \mathrm{C}$ rate.

\section{Conclusion}

An ROM based on the POD method was introduced for simulations of lithium-ion batteries. By means of POD, the dominant patterns of the system dynamics are extracted and named the POMs, which form an optimal subspace on which the most significant information of the state variables is retained. The POD-based ROM is developed by a two-step approximation of the higher-order model; The first approximation arises from discretizing the infinite dimensional governing equations and the second from the truncation of the number of orthogonal modes. The POD-based ROM of lithium-ion battery saves significantly (a speed-up factor of about 7) on computational time and agrees well with the rigorous model. However, the development of the ROM needs either existing experimental data or simulation results of a higher-order model.

\section{Acknowledgment}

The authors gratefully acknowledge the funding support provided by Quallion under the MDA project STTR Phase II: Rechargeable Lithium Ion Battery Operating Life Model.

University of South Carolina assisted in meeting the publication costs of this article.

\section{List of Symbols}



Subscripts

$$
\begin{aligned}
0 & \text { initial condition } \\
\text { eff } & \text { efficient } \\
\text { max } & \text { maximum } \\
\mathrm{n} & \text { negative electrode } \\
\mathrm{p} & \text { positive electrode } \\
\mathrm{s} & \text { separator }
\end{aligned}
$$

Superscripts

$\mathrm{T}$ transpose

\section{References}

1. M. Doyle, T. F. Fuller, and J. Newman, J. Electrochem. Soc., 140, 1526 (1993). 2. T. F. Fuller, M. Doyle, and J. Newman, J. Electrochem. Soc., 141, 1 (1994)

3. W. B. Gu and C. Y. Wang, J. Electrochem. Soc., 147, 2910 (2000).

4. M. Doyle, Y. Fuentes, and J. Newman, J. Electrochem. Soc., 150, A706 (2003).

5. V. Srinivasan and C. Y. Wang, J. Electrochem. Soc., 150, A98 (2003).

6. P. Ramadass, B. Haran, P. M. Gomadam, R. E. White, and B. N. Popov, J. Elec- 
trochem. Soc., 151, A196 (2004)

7. S. Santhanagopalan, Q. Guo, R. Ramadass, and R. E. White, J. Power Sources, 156, 620 (2006).

8. C. Y. Wang, W. B. Gu, and B. Y. Liaw, J. Electrochem. Soc., 145, 3407 (1998).

9. V. R. Subramanian, V. D. Diwakar, and D. Tapriyal, J. Electrochem. Soc., 152, A2002 (2005)

10. S. Liu, Solid State Ionics, 177, 53 (2006).

11. V. R. Subramanian, V. Boovaragavan, and V. D. Diwakar, Electrochem. Solid-State Lett., 10, A255 (2007).

12. B. Bhikkaji and T. Soderstrom, Int. J. Control, 74, 1543 (2001).

13. K. A. Smith, C. D. Rahn, and C. Y. Wang, J. Dyn. Syst., Meas., Control, 2008, 130.

14. D. D. Kosambi, J. Indian Math. Soc., New Ser., 7, 76 (1943).
15. M. Loeve, Acad. Sci., Paris, C. R., 1945, 220.

16. K. Karhunen, Ann. Acad. Sci. Fenn., Ser. A1: Math.-Phys., 1946, 34

17. V. S. Pougachev, Izv. Akad. Nauk SSSR, Ser. Mat., 17, 401 (1953).

18. A. M. Obukhov, Trudy Geofiz. Inst., Akad. Nauk SSSR, 24, 3 (1954).

19. G. Berkooz, P. Holmes, and J. L. Lumley, Annu. Rev. Fluid Mech., 25, 539 (1993)

20. A. Rosenfeld and A. C. Kak, Digital Picture Processing, Academic Press (1982).

21. V. R. Algazi and D. J. Sakrism, IEEE Trans. Inf. Theory, 15, 319 (1969).

22. R. W. Preisendorfer, in Meteorology and Oceanography, Elsevier, New York (1988).

23. L. Sirovich, Q. Appl. Math., 45, 519 (1987).

24. F. Riesz and B. S. Nag, Functional Analysis, Ungar Publishing, NY (1955).

25. K. Kunisch and S. Volkwein, J. Optim. Theory Appl., 102, 345 (1999). 